\title{
Benefits of completing homework for students with different aptitudes in an introductory electricity and magnetism course
}

\author{
F. J. Kontur, K. de La Harpe, and N. B. Terry \\ Department of Physics, United States Air Force Academy, \\ USAF Academy, Colorado 80840, USA
}

(Received 27 May 2014; revised manuscript received 29 October 2014; published 17 February 2015)

\begin{abstract}
We examine how student aptitudes impact how much students learn from doing graded online and written homework in an introductory electricity and magnetism course. Our analysis examines the correlation between successful homework completion rates and exam performance as well as how changes in homework completion correlate with changes in exam scores for students with different physics aptitudes. On average, successfully completing many homework problems correlated to better exam scores only for students with high physics aptitude. On the other hand, all other students showed zero or even a negative correlation between successful homework completion and exam performance. Low- and medium-aptitude students who did more homework did no better and sometimes scored lower on exams than their low- and medium-aptitude peers who did less homework. Our work also shows that long-term changes in homework completion correlated to long-term changes in exam scores only for students with high physics aptitude, but not for students with medium or low aptitude. We offer several explanations for the disparity in homework learning gains, including cognitive load theory, ineffective homework strategies, and various mismatches between homework and exams. Several solutions are proposed to address these possible deficiencies in graded online and written homework.
\end{abstract}

DOI: $10.1103 /$ PhysRevSTPER.11.010105

PACS numbers: 01.40.Fk

\section{INTRODUCTION}

Homework is a key part of nearly every college-level physics course principally because both physics professors and students believe it to be an effective tool for learning physics. That is, doing more homework will lead to greater understanding of physics concepts and increased success on exams. Because of its prevalent use in introductory physics courses, it is not surprising that homework has been one of the most well-studied aspects of physics pedagogy. Numerous articles examined the advantages and disadvantages of online homework [1-5]; other research on homework studied student motivation [6], the deficiency of traditional homework in teaching physics concepts [7], and ways to deal with homework copying [8-10]. Despite the large amount of research that has been done on homework in physics courses, many questions remain about how homework can best be used to aid student learning, including how the benefits of homework depend on student aptitude.

While at least three articles [11-13] touch on this subject, this is the first study which directly examines the question. Cheng et al. found that interactive classes

Published by the American Physical Society under the terms of the Creative Commons Attribution 3.0 License. Further distribution of this work must maintain attribution to the author(s) and the published article's title, journal citation, and DOI. combined with graded online homework led to higher learning gains for all students [11]. Similarly, Kortemeyer et al. found that switching to an online homework system increased course grades [12]. However, these two studies did not directly consider the impact of student aptitude on the effectiveness of homework. Morote and Prichard found that academic background correlated with exam scores but not with homework scores [13], and, while their results suggest that homework and exam scores are not correlated, their work did not directly examine the issue.

The results presented here suggest that incoming student aptitude is a crucial factor in determining how much students learn from doing homework. We characterized the impact of student aptitude by examining how both successful homework completion rates and changes in successful homework completion rates correlate to exam performance for students with different physics aptitude. By characterizing homework effectiveness in these two ways we find that while students with high incoming physics aptitude get some benefit on course exams from completing homework, students with medium and low incoming aptitude get no benefit or even a negative benefit on course exams from completing homework. We examine several possible reasons that homework yields different benefits for students with different physics aptitudes. Finally, we suggest several changes to make homework a more valuable learning tool 
for a wider range of students. As societal needs and public policy push a larger number of students into pursuing science and technology careers, it is important for physics educators to know how beneficial their pedagogical tools are for students having a wide range of abilities. We hope that this investigation will be helpful for teachers dealing with such issues in their classrooms

\section{METHODS}

We begin with a description of the course demographics, content, and homework assignments used during the semesters studied in our research. All students at the United States Air Force Academy (USAFA) take two semesters of calculus-based introductory physics, although only about half of these students major in a science, technical, engineering, or mathematics field. Students generally take mechanics in their freshman year and electricity and magnetism (E\&M) in the fall semester of their sophomore year, unless they had trouble with the prerequisite courses, in which case they typically take $\mathrm{E} \& \mathrm{M}$ in the spring semester. Because of this, the fall semester of E\&M generally has 2-3 times greater enrollment than the spring semester. In this study, we chose to focus on the E\&M course rather than the mechanics course for several reasons-students have had less high-school exposure to the material, it is more challenging than the mechanics course, and, being later in the course sequence, we have more grade data from prerequisite courses to characterize student aptitude.

The E\&M course is taught in sections where the enrollment is set at approximately 20 students, with between 5 and 12 different instructors teaching the course each semester. All sections of the course use the same textbook, use the same syllabus, complete the same assignments, and take the same quizzes and exams. During the semesters considered in this study, the E\&M course textbook was Essential University Physics by Richard Wolfson [14,15]. All USAFA physics instructors are trained to use a variety of interactive teaching techniques, including just-in-time teaching (JiTT) [16], peer instruction [17], think-pair-share [18], and board work problem solving.

Homework, consisting of 2-3 book problems for each lesson, was initially administered written, and in recent semesters online through the Mastering Physics online system. For Mastering Physics homework, students were given up to 5 tries to get the correct answer on homework problems, with no deduction for an incorrect answer until the final attempt. The amount of credit given for homework varied from a low of $6.0 \%$ to a high of $9.6 \%$ of the total course grade. Homework was generally due each lesson or each week to discourage cramming. For the purposes of this study, when considering online homework, we used the Mastering Physics homework correctness score as the successful homework completion score.
There were three midterms and a cumulative final exam for all but one of the semesters of E\&M studied. The midterms were each worth $\sim 10 \%$ of the course grade and the final exam was worth 25\%. In the spring 2009 E\&M course, there were only two midterms, which were each worth $13.5 \%$ of the course grade; the final exam was worth $25 \%$. The midterms were 80 - to 110 -min in-class exams comprised of ten conceptual multiple-choice questions worth $50 \%$ of the exam points and two or three homework-type workout problems worth $40 \%-50 \%$ of the exam points (8 of the 20 midterms also had a short-answer question that was worth $10 \%$ of the exam points). The final exam consisted of 30-35 conceptual multiple-choice questions, worth $60 \%-70 \%$ of the exam points, and two or three workout problems, worth $23 \%-36 \%$ of the exam points. The final exam sometimes had a short answer question as well, worth between $6 \%$ and $17 \%$ of the exam points. In addition to exams and homework, the remainder of the course points was a mix of grades on student journals, JiTT assignments, and short in-class quizzes.

The first drafts of the exams were written by personnel at USAFA's Center for Physics Education Research. These personnel were not instructors in the E\&M course, and they wrote the exams using the course learning objectives. The exams were edited based on feedback from instructors. In general, the editing process involved rewording questions to make them more understandable and replacement of questions based on which concepts were emphasized or not emphasized in class. The overall goal throughout the exam writing and editing process was to test physics understanding rather than memorization or pattern matching.

In this study, course exams were used to measure student learning rather than research-based concept tests like the Force Concept Inventory (FCI) [19], the Conceptual Survey of Electricity and Magnetism (CSEM) [20], or the Brief Electricity and Magnetism Assessment [21]. The first reason for using course exams is that light and optics (a quarter of the content in our E\&M course) is not covered on the aforementioned concept tests. The second reason is that the concept tests assess conceptual knowledge rather than problem-solving ability. While it has been shown that increased conceptual knowledge leads to better problem solving [17], we wanted to separately identify if homework was benefiting students' conceptual and/or problemsolving abilities. In this study, a group of students is said

TABLE I. We divided students into four different physics aptitude groups according to their GPA in calculus 1, calculus 2 , and mechanics.

\begin{tabular}{lc}
\hline \hline GPA & Physics aptitude group \\
\hline $4.00-3.25$ & High \\
$3.24-2.50$ & Medium-high \\
$2.49-1.75$ & Medium-low \\
$1.74-1.00$ & Low \\
\hline \hline
\end{tabular}




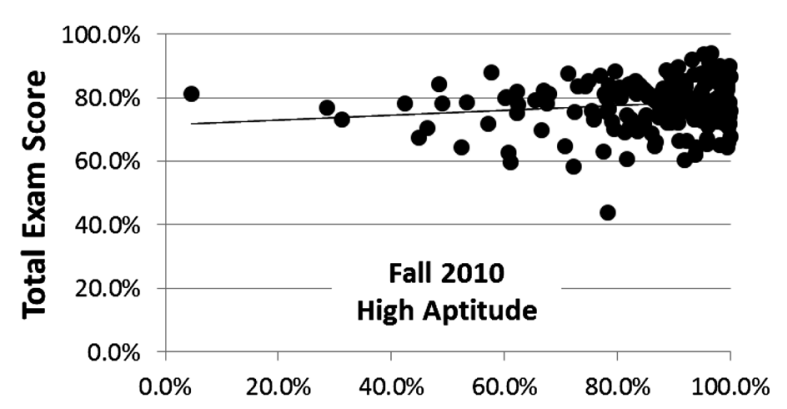

Homework Completion Score
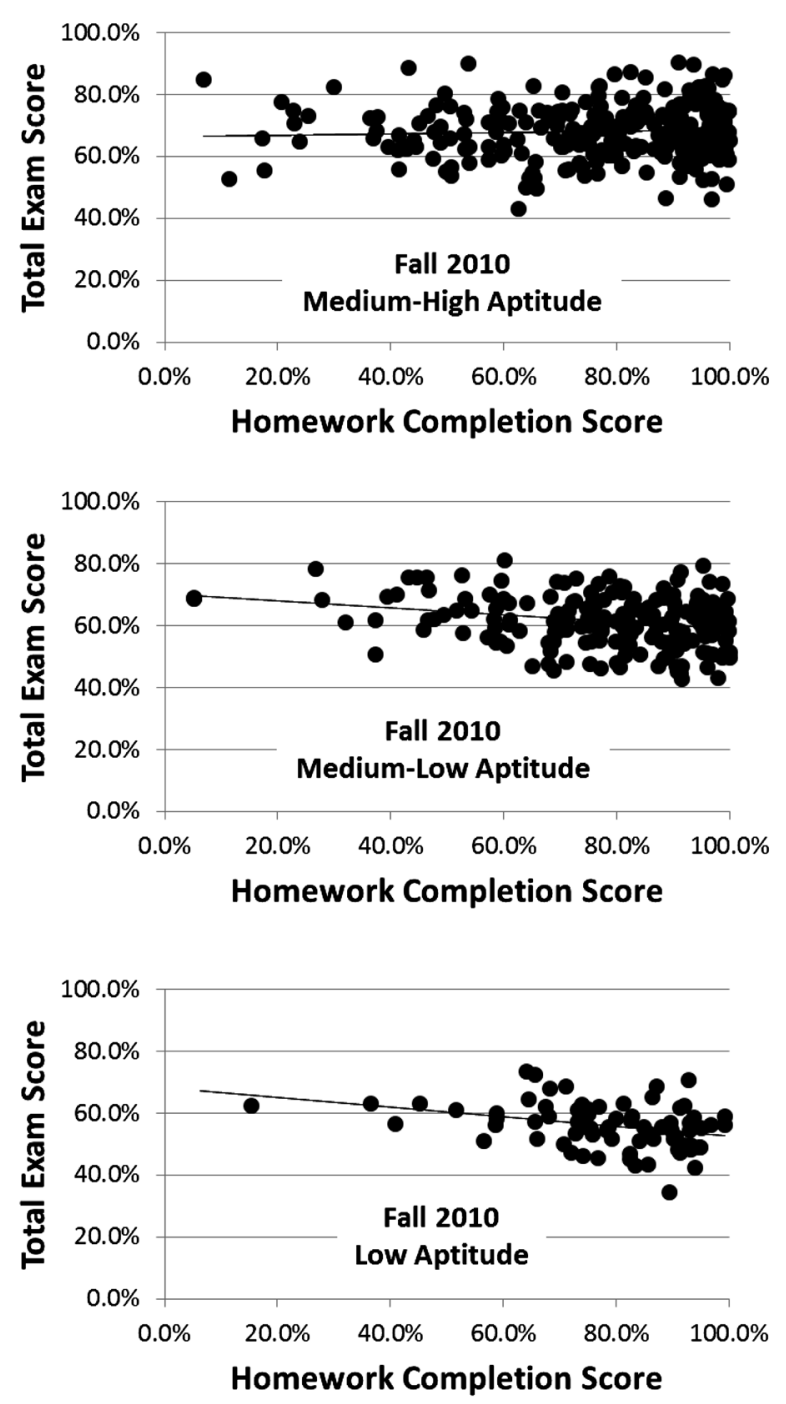
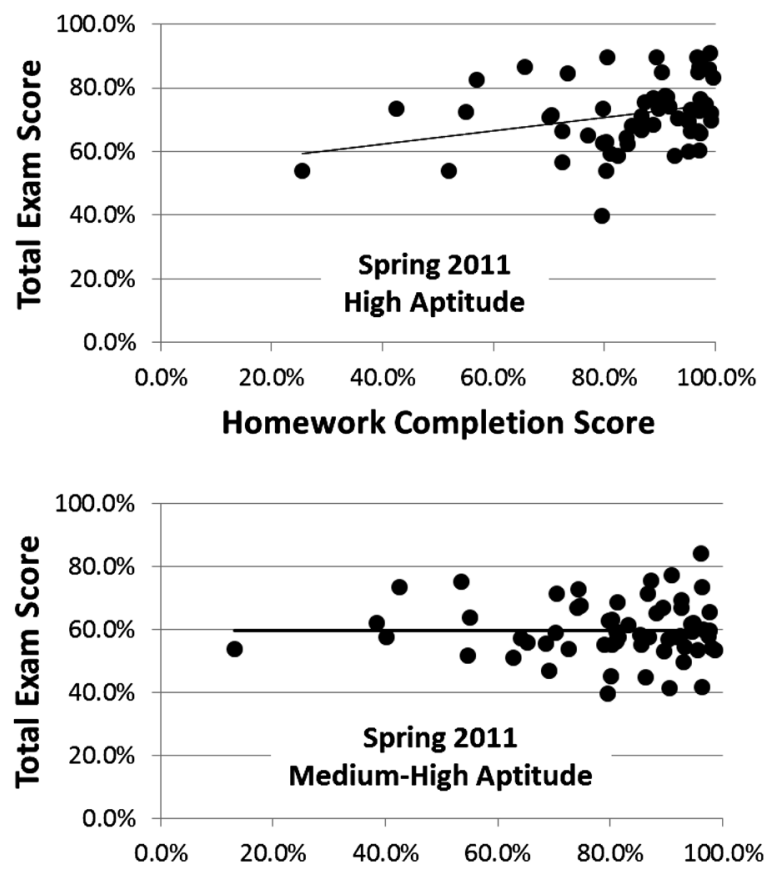

Homework Completion Score
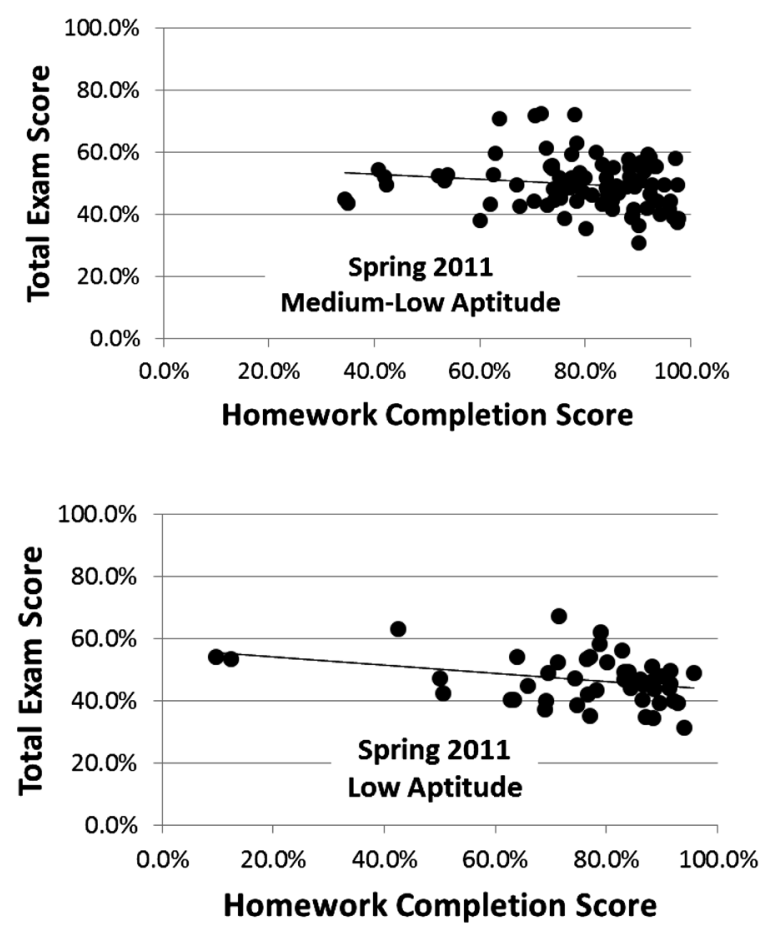

FIG. 1. The weighted-average score on all exams vs homework completion score for students in different aptitude groups who took the USAFA E\&M course in fall 2010 (left plots) and spring 2011 (right plots). Line fits are included with the data. The semesters shown here are representative of the correlations of the other 7 semesters studied.

to benefit from doing homework if there was a positive correlation between homework scores and exam scores.

We determined physics aptitude by identifying the variable that, at the beginning of the semester of the E\&M course, best predicted student success on E\&M exams. While there were positive correlations between grades and SAT math scores, ACT math and science scores, FCI pre- and post-test scores, and CSEM pretest scores $\left(r^{2}=0.14-0.31\right)$ the strongest predictor of exam scores were students' combined grade point averages (GPA) in three incoming prerequisite courses (calculus 1 and 2 and mechanics), with $r^{2}=0.52$. The high correlation 
between calculus grades and E\&M exam performance is consistent with previous studies that found a high correlation between students' math skills and their exam grades in college physics [22].

Students' combined GPA in calculus 1 , calculus 2 , and mechanics was used to define student's aptitudes. The highest GPA that can be achieved is 4.0, for a student who received an A in all three courses. The lowest GPA is 1.0 , for a student who received a D in all three courses. A student who fails one of the courses must retake it before taking E\&M. For simplicity, in the case of students who took a course more than once, we only considered their most recent grade in the course. As shown in Table I, we grouped students into four physics aptitude groups based on their GPAs in the three prerequisite courses. Each aptitude group covers 0.75 grade points, from the maximum of 4 to the minimum of 1 . For the remainder of this article, the aptitude groups will be referred to using their names in Table I.

\section{RESULTS}

In our analysis, we examine the correlation between successful homework completion and exam scores for each aptitude group described in the previous section. We also consider the correlation between long-term changes in successful homework completion rates and long-term changes in exam performance for each aptitude group. Before we continue, it is worth noting that we as instructors assign homework to our students because we believe that all students will receive a learning benefit from doing homework. This belief is predicated on the idea that there is a strong positive correlation between making an honest effort to do well-chosen homework problems and student learning, qualities we attempt to measure using homework scores and exam scores. If there is no correlation between successful homework completion and exam scores, we should carefully rethink these assumptions, especially considering that assigning, completing, and grading homework is often a very time-intensive activity for both students and teachers. The remainder of this section is devoted to examining the strength of the expected correlation between homework scores and learning for all of our students.

Figure 1 plots the total exam score (the average, weighted by percentage of overall course grade, of the scores on the midterm exams plus the final exam) vs homework completion score (as mentioned in Sec. II, this is the same as the Mastering Physics correctness score) for each student who took E\&M in the fall 2010 and spring 2011 semesters. Included with the plots are line fits for the data. As mentioned in Sec. II, the fall semesters of E\&M have 2-3 times greater enrollment than the spring semesters, so there is a larger set of data for the fall 2010 semester compared to the spring 2011 semester. Remarkably, medium-low- and low-aptitude students have a negative benefit from doing homework for both semesters shown in Fig. 1. This indicates that, on average, the more homework that medium-low- and low-aptitude students did, the worse they performed on exams.

A visual inspection of Fig. 1 might suggest that students completing less than $50 \%$ of the homework are the dominant influence on the slope of the graphs and that removing them from the analysis would make the slopes positive, especially for the low- and medium-low-aptitude students. However, removing the $6 \%$ of students who successfully completed less than $50 \%$ of the homework from the analysis only changes the slope of the graph for the low- and medium-low-aptitude students by about $1 \%$. The slopes are still slightly negative, and certainly not strongly positive as predicted by our common sense notions of homework. On the other hand, removing the 6\%-12\% of students who successfully completed less than $50 \%$ of the homework from the analysis increases the slope of the graph by about $4 \%$ for the medium-high- and the high-aptitude students. This is at least consistent with (or perhaps even strengthens) our conclusion that homework appears to be "working" for our best students, but not for our struggling students. The negative benefit effect for medium-low- and low-aptitude students occurred in 6 of the 7 semesters analyzed in this study.

Figure 2 summarizes the results of Fig. 1 by illustrating the average benefits of successful homework completion realized by students of various aptitudes during the 7 semesters we studied. Each semester of the 7 semesters is first analyzed independently and then combined (rather than simply grouping all the semesters together for a single analysis) to control for variability between exams from semester to semester. This way the learning activity (homework) for each semester is only compared against the learning measurement (exams) for that semester. The error bars show the standard deviation of the slopes from the 7 semesters. Figure 2 shows that, on average, medium-lowand low-aptitude students had a significant, consistent, and

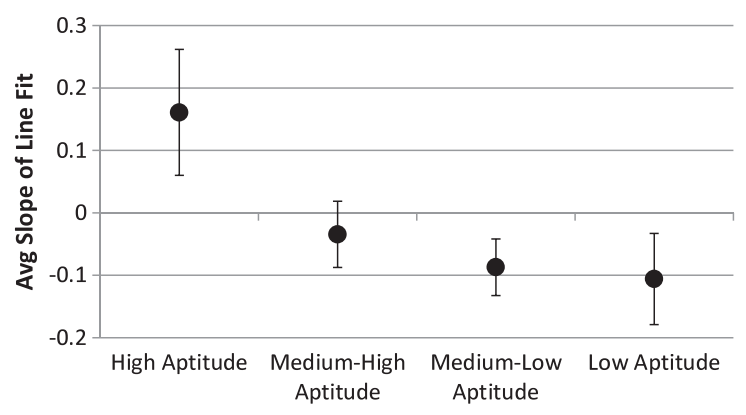

FIG. 2. Average of the slopes of the line fits between successful homework completion and exam score for four different aptitude groups. The data are from 7 semesters of E\&M when homework was administered online and graded only for correctness. Error bars are the standard deviations of the slopes for the 7 different semesters. 


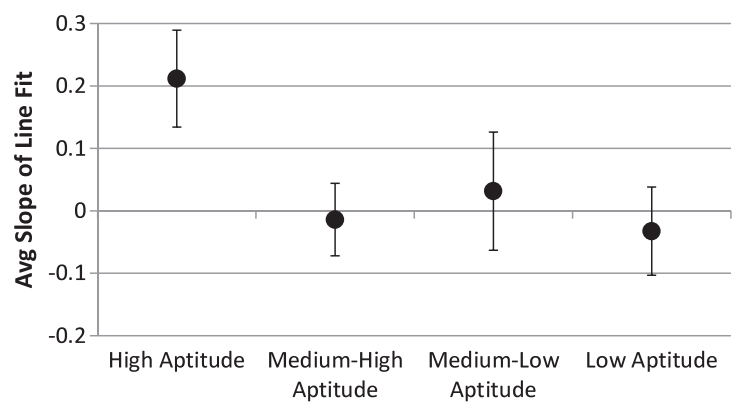

FIG. 3. Average of the slopes of written homework scores vs total exam score plots as a function of student aptitude for four semesters when homework problems were administered in a graded, written format. Error bars are the standard deviations of the slopes for the 4 different semesters where written homework was used.

repeatable negative benefit from completing homework. The results in Fig. 2 are almost identical if the conceptual multiple choice question portions of the exam and the homework-style workout problems on the exam are considered separately.

Prior to spring 2009, the USAFA E\&M courses often employed a combination of instructor-graded written homework and computer-graded Mastering Physics homework. In some semesters, the written homework would be students' solutions to original problems written by the lead teacher for the course, and, in other semesters, the written homework would be students' written solutions to the Mastering Physics problems. Regardless, homework data from those semesters should indicate if written homework is more effective than online homework for lower aptitude students. The specific semesters we examined were the fall 2006-spring 2008 semesters of E\&M. Similar to the analysis in Fig. 2, we graphed written homework score vs total exam score for different aptitude students and determined the slopes of those plots. Taking the average slopes from those four semesters yields the data shown in Fig. 3. The data show that, in fact, the negative benefits seen in Fig. 2 are no longer present for the written homework. However, it appears that written homework is only effective for high-aptitude students. For all other students, homework had essentially no effect on total exam scores. We assign homework because we expect there is a causative relationship between homework and learning. The correlations in Figs. 2 and 3 suggest that this anticipated causative relationship should be reevaluated for major segments of our student population.

Besides determining the correlation between total homework completion and test scores, we also examined a second common sense prediction that students who change their homework behavior by increasing the amount of homework they do will generally benefit. To test this prediction, we examined the changes in homework completion rates of students who took mechanics and E\&M in successive semesters. For each student in E\&M, we
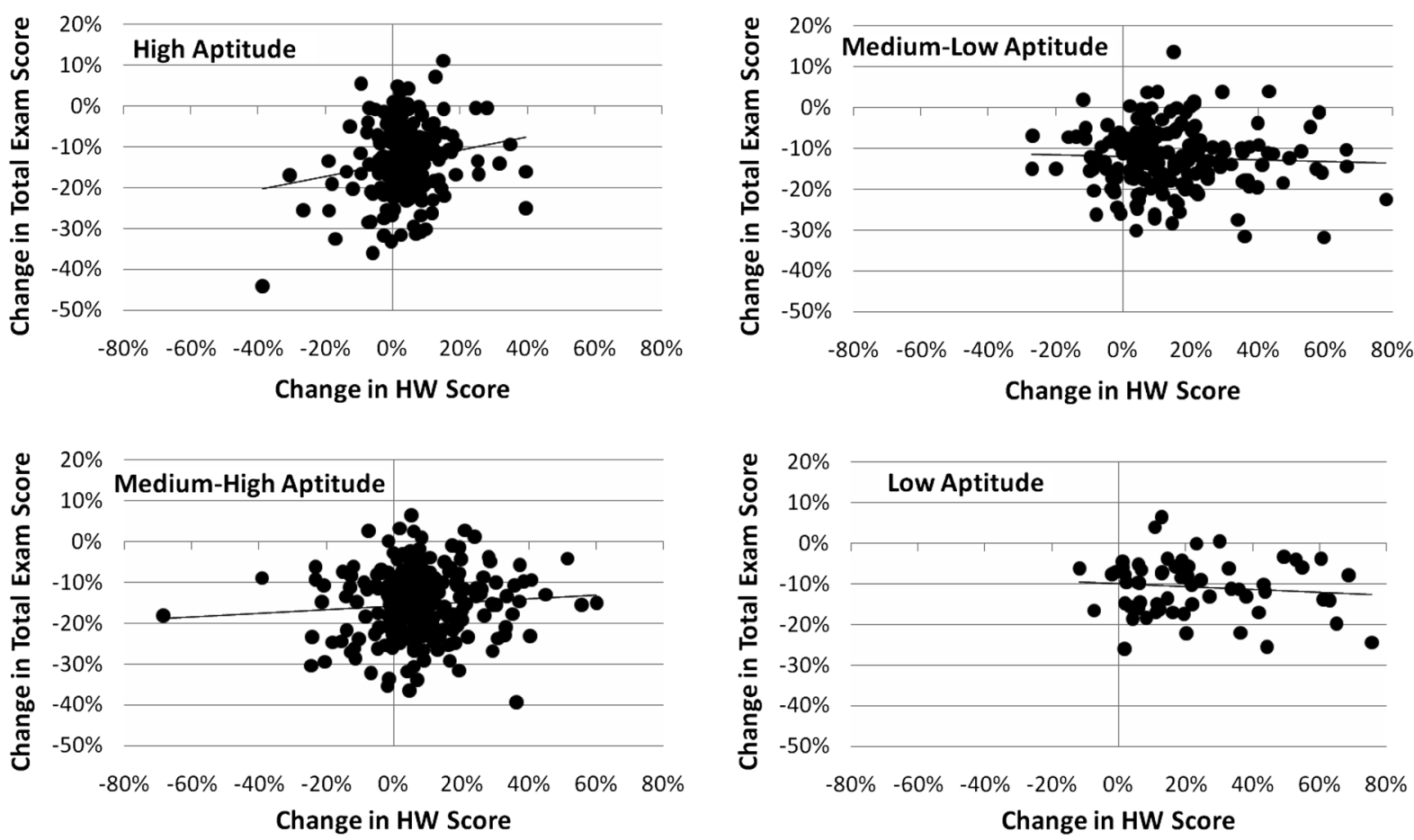

FIG. 4. Plots of the change in the total exam score vs the change in homework score for students moving from spring 2009 mechanics to fall 2009 E\&M. Each plot shows students in different aptitude groups. Line fits are included with the data. These results shown here are typical of all the semesters we examined. 


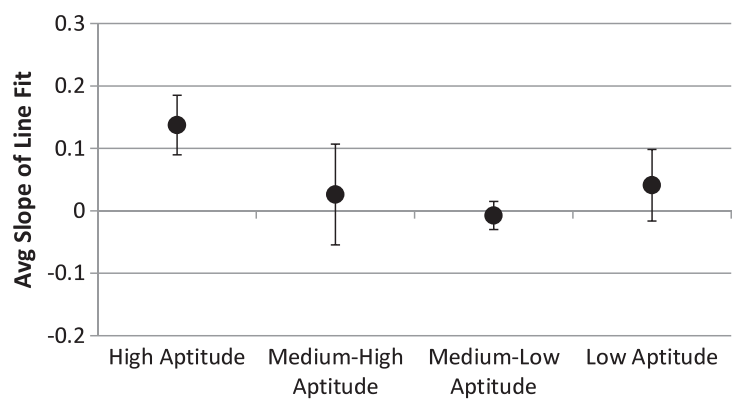

FIG. 5. The average correlation between the change in HW score vs change in total exam score from mechanics to E\&M as a function of student aptitude for 5 semesters of E\&M being studied. Error bars are the standard deviations of the slopes for the different semesters.

measured the change in successfully completed homework between the two courses and compared this to the change in total exam scores for each student. We were only able to examine 5 of the 7 semesters because it was not possible to determine the change in homework score for two semesters where homework was ungraded in the mechanics course. Figure 4 shows the change in exam scores as a function of the change in homework scores for students who took mechanics in spring 2009 and E\&M in fall 2009. The average slopes of the changes in homework vs change in homework for all 5 semesters are shown in Fig. 5. Again, we see that high aptitude students show a consistent benefit from doing more homework but other students do not.

While Figs. 4 and 5 examine changes in student behavior changes between mechanics and E\&M, we also examined changes in student behavior within the E\&M course. Figure 6 looks at the change in individual exam scores, from one exam to the next, as a function of the change in homework score, from one exam to the next within a semester of E\&M. The graph labeled midterm 2 plots the change in exam score from midterm 1 to midterm 2 as a function of the change in homework score from course block 1 to course block 2 . Similarly, the graph labeled midterm 3 plots the change in exam score from the average of midterms 1 and 2 to midterm 3 as a function of the change in the average homework score from course blocks 1 and 2 to course block 3 .

Figure 5 shows that increasing homework completion from mechanics to E\&M only benefits high-aptitude students on exams, which is consistent with the other data presented thus far. However, though the data are noisy, Fig. 6 shows that increasing homework completion before an exam results in better performance, on average, on that exam for nearly all students. In particular, medium-lowaptitude students clearly show positive benefits from increasing their rate of homework completion in the lessons leading to an exam. This is likely why students have such a strong belief that homework is beneficial. They can see immediate benefits, on the next midterm, when they do more homework. Unfortunately, Fig. 5 shows that a long-term increase in homework completion does not correspond to a long-term increase in learning for low- or medium-aptitude students. Instead, the benefits due to increasing homework effort appear to only yield short-term rather than long-term benefits for all but our best students.

\section{DISCUSSION}

While instructors assign homework because we believe all of our students will learn from doing it, the data presented in the previous sections contradict this belief, instead suggesting that homework is an effective learning tool only for our best students. It is important to note that correlation does not imply causation, meaning that the data do not tell us why homework doesn't work as expected. If our low-aptitude students are getting something out of homework, it is clearly not what we expect them to learn. There are a number of possible explanations for why homework does not work for our struggling students, ranging from how students approach problems, what problems we assign, the sequencing of homework problems, the ways we use to motivate homework, how lessons
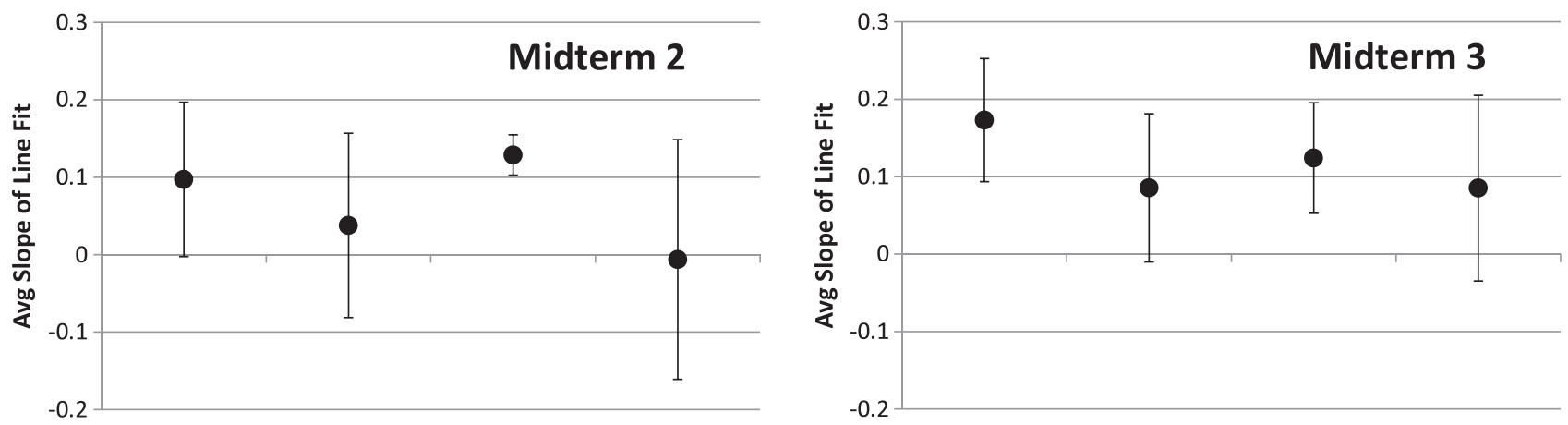

FIG. 6. Average slopes of line fit data of change in homework score vs change in exam score (left) from midterm 1 to midterm 2 , and (right) from midterms 1 and 2 to midterm 3. Only 6 of the 7 semesters being studied are included in this analysis, with spring 2009 left out because there were only 2 midterms during that semester. 
couple with follow-up homework, and how exams measure what is learned by doing homework, to name a few. Fully understanding the problem of why homework is only effective for our high-aptitude students is an area where significant productive research remains to be done.

We begin this discussion by considering three possible reasons that homework is ineffective: students are not working the homework problems in an effective manner, our exams are not appropriately measuring student learning, or we are not assigning the right problems. However, we are not proposing any of these ideas as a causative explanation based on the data presented in this article. Our major finding is that, even though most teachers do think there is a connection between doing more homework and doing better on exams, our analysis finds that no such connection exists for most students.

\section{A. Homework copying}

We begin by considering the prevalence of copying $[8,9]$, which is often an attempt to save time and effort and can range from blatant cheating to a misguided over reliance on solution manuals. To characterize the levels of homework copying in our course, we went through 19 different problem sets of Mastering Physics for the fall 2010 semester and measured the number of attempts used by randomly selected low- and high-aptitude students. We then determined the ease with which these students did the homework by dividing their homework score by the number of attempts they used. We excluded students who did less than $50 \%$ of the homework so they would not skew the data. There was no statistically significant correlation between the number of points students earned per attempt and their overall exam score. We also found no correlation between test scores and the amount of time students were logged into the Mastering Physics homework server. Finally, we note that anonymous surveys of student homework behavior showed that less than $6 \%$ of students self-reported using on-line solutions to complete homework. These findings suggest that copying does not explain why homework is ineffective for our struggling students.

\section{B. Student approaches to doing homework}

Having ruled out copying as an explanation for why homework is ineffective for some groups of students, but not others, we turn our attention to novice approaches to homework. For many students, the key to doing homework problems is finding the right equation. Once the right equation is found, all that is necessary is to plug-and-chug to get the correct answer. Unfortunately, while this method of doing homework may teach students how to do that specific problem, it does not teach them what concept or concepts underlie the problem and how the concepts in the problem might connect to concepts that they have learned previously (see p. 38 of Ref. [18] for a visual representation of this phenomenon). As Redish et al. stated: "We are frustrated by the tendency many students have to... spend a large amount of time memorizing long lists of uninterpreted facts or performing algorithmic solutions to large numbers of problems without giving any thought or trying to make sense of them" [23].

Unfortunately, simply telling students not to use ineffective strategies probably won't solve the problem. Instead, we as instructors may need to teach them effective homework strategies to replace the ineffective strategies. The first step to doing this is to identify effective strategies. This is more challenging than it may seem, because our data suggest that learning strategies that are effective for high-aptitude students may not be equally effective for medium- and low-aptitude students. Different strategies may be useful for these latter two groups. Identifying and characterizing the strategies favored by and useful for each aptitude group is the subject of a future article.

\section{Validity of course exams in assessing learning}

Just as it is important to consider the strategies our students employ to complete their homework, it is also important to consider how we measure the learning gains for our various students. Our exams are reviewed by multiple physics instructors both affiliated and not affiliated with the course. One of the principal things considered in reviewing the exams is whether it is properly assessing the course objectives, which is also the basis for the homework and other learning exercises in the course. Our exams consist of several problems for students to solve, some of which students have seen before and some which are subtly different than what they have seen before. We also administer a series of multiple-choice questions that probe student understanding of electricity and magnetism concepts. From this perspective, our exams have face validity and are probing the concepts and skills we are trying to teach our students.

On the other hand, it is quite possible students with low incoming grades in prerequisite courses are learning something from homework, but what they are learning are basic math and science skills, rather than the higher level physics conceptual and problem-solving skills probed by our exams. However, their high-aptitude peers have already learned the basic skills and instead can focus on learning advanced concepts and problem-solving skills from doing homework. While successful completion of exam problems demands a mastery of algebra, for example, our exams are not designed to accurately differentiate between levels of algebra mastery. Perhaps the optimum balance of simple homework problems that review basic skills and homework problems on more advanced topics is different for high- and low-aptitude students. For example, conceptual questions on electric forces that are designed to probe students' understanding of electric fields also require a basic understanding of forces. Perhaps students completing homework 
problems dealing with electromagnetic forces are able to solidify their understanding of the basic idea of Newtonian forces. Understanding of Newtonian forces is not well differentiated by conceptual questions about electromagnetic forces, meaning a student is penalized for not learning about electric forces and remains unrewarded for gains in their understanding of Newtonian forces. Our exams may simply not measure gains in fundamental skills which we assumed (perhaps erroneously) that students have previously mastered, even if these gains in fundamental skills are very large.

\section{Cognitive load}

Having considered effective homework strategies and what our exams are actually measuring, the final possibility we consider is that there is something intrinsically inappropriate with the homework problems that we give our students for practice. There are a number of interconnected parameters which might impact the effectiveness of homework, including the number of assigned problems, sequencing, difficulty level, student motivation, instructor feedback, grading practices, etc. For example, while exams require students to solve a particular problem selected from a group of topics, homework problems are often linked to a particular lesson which only covers one or two physics concepts. While exams require students to remember concepts and problem solving strategies, homework assignments typically allow students to use their textbook, notes, and other sources. Since it would be difficult to consider all of these variables in a single study, we instead discuss principles that explain the effectiveness of homework as a function of student aptitude.

One principle which can explain differential learning gains is cognitive load theory, which posits that learners can simultaneously process only a limited number of ideas in their working memory [24,25]. Additional processing power is available from long-term memory, provided that students have that knowledge encoded in long-term memory. Knowledge thus stored in long-term memory is known as a schema. High-aptitude students appear to possess a large number of useful schemas, both from their previous courses and from an ability to better incorporate early course material into new schemas. As homework problems become more complicated, high-aptitude students who call upon these schemas can supplement their limited working memory, enabling them to process the homework and use it to learn new material.

On the other hand, low-aptitude students possess only a limited number of schemas from previous courses and it is possible that some of these schemas may even be incorrect. Thus, low-aptitude students can only supplement their working memory with a very small number of correct schemas. In some cases, low-aptitude students may build schemas associated with basic skills that instructors already expect students to know. On the other hand, low-aptitude students may regularly experience cognitive overload when dealing with complicated problems. Students may deal with this cognitive overload by creating new schemas for each homework problem, schema which may focus on surface features of the problem rather than on deep physics.

Inevitably, the inefficient schema building that occurs for low-aptitude students while doing homework problems hinders their success on exams. This will have a cumulative effect, because a proper understanding of physics requires students to build a coherent framework of concepts during the semester. A student who has separate, often incorrect, schema for all of the different homework problems (based on surface features, for example) will have an increasingly difficult time recognizing the conceptual framework that is being built over the semester. Therefore, though they might be able to memorize and/or pattern match enough to do adequately on an exam that tests a limited number of concepts, low-aptitude students will fail to achieve the big picture understanding that is necessary to do well on a more comprehensive test, such as the final exam. We expect that, for our medium-aptitude students, knowledge in long-term memory would sufficiently supplement their working memories to prevent cognitive overload for some problems and not others. They would therefore learn from some homework problems and not others, making their learning gains somewhere between those of the highaptitude and the low-aptitude students.

\section{E. Possible solutions}

Before we suggest solutions to the aforementioned difficulties, we note both cognitive load theory and the idea that struggling students only learn basic skills from homework both explain the differential learning gains between students of various aptitudes. These ideas are interconnected. We highlight this point to emphasize that student preparation is the main problem rather than specific systems of homework. A possible solution to the issue of homework placing an excessive cognitive load on low-aptitude students is to assign easier homework problems which focus on fundamental skills. Teoderescu et al. attempted this with preclass work [26]. Their results indicate "that choice allows students to earn a reasonable amount of points with problems that match their ability." However, they also found that, for more difficult problems, "C and D [students] may simply elect not to invest more time when their success rate drops, even when sufficient points have not been accumulated." The issue with this is that easier homework problems may leave students ill prepared for exams testing advanced skills. Another option is strengthening prerequisite course requirements [27].

Another possible solution that has met with some success in previous studies is to require mastery of prerequisite knowledge before a student can move on 
to the next learning topic. This would require the students to achieve the level of understanding of high-aptitude students before moving from mechanics to $\mathrm{E} \& \mathrm{M}$ or before moving from one section of the E\&M course to the next. One study of an E\&M course that employed mastery learning found that students who went through the course did $10 \%-15 \%$ better on the final exam compared to equivalent students in a traditional course [28]. In addition, on a retest of the course material given two months later, students who were in the mastery learning course did 15\%-20\% better than students who took the traditional course, indicating that not only did the students learn more in the mastery learning course, they retained what they learned for a longer period of time. In addition, a meta-analysis of mastery learning courses found that the average improvement of high-aptitude students in such courses is 0.40 standard deviations while the average improvement of low-aptitude students is 0.61 standard deviations [29]. This is consistent with our findings that while high-aptitude students benefit from doing homework, struggling students do not get much benefit from homework. If low-aptitude students are required to demonstrate mastery of each subject before moving on, not only will they start each new section of the course with more prerequisite knowledge, they should also get more out of the time that they put into homework and quite possibly other course learning activities.

One final suggestion is that in order to make the skills practiced in homework better match the skills required for success on exams, instructors can make the homework more like exams. In a review of research on learning, Rohrer and Pashler found that "a combination of study and tests is more effective than spending the same amount of time reviewing the material in some other way" [30]. In addition to the learning advantages of testing vs reviewing, Rohrer and Pashler also discuss a technique called interleaving [30]. In interleaving, learning activities are not grouped together by subject. Rather, several different subjects are mixed together in the learning activities. So, for example, if Faraday's law is covered in a particular lesson, the homework for that lesson could have Coulomb's law and circuit analysis and magnetic force problems in addition to Faraday's law problems. They cite one study where mathematics test scores increased by a factor of 3 when interleaving was employed [31]. As discussed above, one of the skills required for success on exams that is not typically practiced on homework is identifying which concept applies to a particular problem. Interleaved homework remedies this issue.

\section{SUMMARY AND CONCLUSIONS}

This paper examined the effectiveness of online and written homework as a function of student aptitude. We studied the effectiveness of homework by determining the correlation between successful homework completion and exam scores and the correlation between changes in homework completion and changes in exam scores. We were surprised to find that only high-aptitude students seemed to derive any measurable benefit from completing homework, while all other students received zero or even negative benefit from doing homework. This result is troubling because doing homework is typically considered a key to success in physics courses, so much so that struggling students are often advised to do more homework in order to be better prepared for exams. Our findings indicate that this learning strategy may be completely ineffective for most of our students.

We explained these results in terms of student preparation and argued that the results are not due to a particular way of administering homework but rather arise from the fact that poor fundamental skills in mathematics or Newtonian mechanics prevent students from learning the more advanced skills that are the focus of an introductory electricity and magnetism course. Homework imposes an excessive cognitive load on low-aptitude students and sometimes even mediumaptitude students. For low-aptitude students, this may encourage a focus on surface features of homework problems rather than deeper learning. We also discussed how homework may help struggling students learn basic skills rather than more advanced skills and how improvements in basic skills may not be measured by course exams. Last, we suggested several solutions to address these deficiencies in homework, including employing mastery learning in physics courses and interleaving multiple concepts in homework assignments. Several of these suggestions should be fairly easy to include in most introductory physics courses. Based on the evidence presented in this article, making such changes to homework could lead to significant learning gains.

\section{ACKNOWLEDGEMENTS}

We would like to thank Dr. Lauren Scharff in the Scholarship of Teaching and Learning Center at the United States Air Force Academy for her support and guidance in this research. 
[1] J. Mestre, D. M. Hart, K. A. Rath, and R. Dufresne, The effect of web-based homework on test performance in large enrollment introductory physics courses, J. Comput. Math. Sci. Teach. 21, 229 (2002).

[2] S. W. Bonham, D. L. Deardorff, and R. J. Beicherner, Comparison of student performance using web and paperbased homework in college-level physics, J. Res. Sci. Teach. 40, 1050 (2003).

[3] A. M. Pascarealla, The influence of web-based homework on quantatative problem-solving in a university physics class, in Proceedings of the NARST Annual Meeting, (National Association for Research in Science Teaching, Vancouver, BC, Canada, 2004).

[4] R. Warnakulasooriya and D. Pritchard, Learning and problem-solving transfer between physics problems using web-based homework tutor, in Proceedings of the World Conference on Ed. Multimedia, Hypermedia and Telecommunications, (Association for the Advancement of Computing in Education (AACE), Montreal Canada, 2005).

[5] G. Kortenmeyer, M. Hall, J. Parker, B. Minaei-Bidgoli, G. Albertelli, W. Bauser, and E. Kashy, Effective feedback to the instructor from online homework, J. Asynchronous Learn. Networks 9, 19 (2005).

[6] F. J. Kontur and N. B. Terry, Motivating students to do homework, Phys. Teach. 52, 295 (2014).

[7] E. Pak and S. J. Kim, Students do not overcome conceptual difficulties after solving 1000 traditional homework problems, Am. J. Phys. 70, 759 (2002).

[8] D. J. Palazzo, Y. J. Lee, R. Warnakulasooriya, and D. E. Pritchard, Patterns, correlates, and reduction of homework copying, Phys. Rev. ST Phys. Educ. Res. 6, 010104 (2010).

[9] M. Grams, Cramster, friend or foe?, Phys. Teach. 49, 225 (2011).

[10] M. Grams, The cramster conclusion, Phys. Teach. 49, 291 (2011).

[11] K. K. Cheng, B. A. Thacker, R. L. Cardenas, and C. Crouch, Using an online homework system enhances students' learning of physics concepts in an introductory physics course, Am. J. Phys. 72, 1447 (2004).

[12] G. Kortenmeyer, E. Kashy, W. Benenson, and W. Bauer, Experiences using the open-source learning content management and assessment system LON-CAPA in introductory physics, Am. J. Phys. 76, 438 (2008).

[13] E. Morote and D. Pritchard, Technology closes the gap between students' individual skills and background differences, in Proceedings of the 15th International Conference Society for Information Technology \& Teacher Education, (Association for the Advancement of Computing in Education (AACE), Atlanta, GA, 2004).

[14] R. Wolfson, Essential University Physics, 1st ed. (Addison Wesley, San Franscisco, 2007).
[15] R. Wolfson, Essential University Physics, 2nd ed. (Addison Wesley, San Francisco, 2012).

[16] G. M. Novak, E. T. Patterson, A. D. Gavrin, and W. Christian, Just-in-Time Teaching: Blending Active Learning with Web Technology (Prentice Hall, Upper Saddle River, NJ, 1999).

[17] E. Mazur, Peer Instruction (Prentice Hall, Upper Saddle River, NJ, 1997).

[18] R. D. Knight, Five Easy Lessons: Strategies for Successful Physics Teaching (Addison Wesley, San Francisco, 2004).

[19] D. Hestenes, M. Wells, and G. Swaekhamer, Force Concept Inventory, Phys. Teach. 30, 141 (1992).

[20] D. P. Maloney, T. L. O'Kuma, C. J. Heiggelke, and A. V. Heuvelen, Surveying students' conceptual knowledge of electricity and magnetism, Am. J. Phys. 69, S12 (2001).

[21] L. Ding, R. Chaba, B. Sherwood, and R. Beichner, Evaluating an electricity and magnetism tool: brief electricity and magnetism assessment, Phys. Rev. ST Phys. Educ. Res. 2, 010105 (2006).

[22] D. E. Meltzer, The relationship between mathematics preparation and conceptual learning gains in physics: A possible 'hidden variable' in diagnostic pretest scores, Am. J. Phys. 70, 1259 (2002).

[23] E. F. Redish, J. M. Saul, and R. N. Steinberg, Student expectations in introductory physics, Am J. Phys. 66, 212 (1998).

[24] J. Sweller, Cognitive load during problem solving: Effects on learning, Cogn. Sci. 12, 257 (1988).

[25] F. Paas and P. Ayres, Cognitive load theory: A broader view of the role of memory and learning in education, Educ. Psychol. Rev. 26, 191 (2014).

[26] R. Teodorescu, D. Seaton, C. Cardamone, S. Rayyan, J. Abbott, A. Barrantes, A. Pawl, and D. Prictchard, When students can choose easy, medium, or hard homework problems, in Physics Education Research Conference 2011, Omaha, Nebraska, 2011.

[27] N. B. Terry, K. d. L. Harpe, and F. Kontur, The evolution of the learning gap between students with strong prerequisite skills and students with weak prerequisite skills, J. College Sci. Teach. (to be published).

[28] S. M. Austin and K. Gilbert, Student performance in a Keller-Plan course in introductory electricity and magnetism, Am. J. Phys. 41, 12 (1973).

[29] C.-L.C. Kulik, J. A. Kulik, and R. L. Bangert-Downs, Effectiveness of mastery learning: a meta-analysis, Rev. Educ. Res. 60, 265 (1990).

[30] D. Rohrer and H. Pashler, Recent research on human learning challenges conventional instructional strategies, Educ. Res. 39, 406 (2010).

[31] D. Rohrer and K. Taylor, The shuffling of mathematics problems improves learning, Instrumentation Science and Technology 35, 481 (2007). 